\title{
Situación educativa en Colombia entre 1950 y 1980 y su relación con el papel del maestro
}

Por: Lina Marcela Moreno Triana ${ }^{1}$

"Para los tiempos que vienen, no soy yo el maestro que debéis elegir, porque de mí sólo aprenderéis lo que tal vez os convenga ignorar toda la vida: a desconfiar de vosotros mismos"

Antonio Machado

El siguiente ensayo surge de las lecturas y debates realizados en el componente Políticas y Legislación en Colombia, pensando en la forma como los maestros han adquirido una determinada postura en unos momentos y contextos específicos y su relación con las dinámicas educativas en Colombia.

Es así como la educación en Colombia se ha visto mediada por una serie de procesos políticos, económicos, sociales, entre otros, que se ubican dentro de un espacio contextualizado, es decir, la educación va de la mano con una serie de hechos históricos que la enmarcan dentro de una realidad y de esta forma la situación educativa en Colombia fue, ha sido y será una forma que se sale de la constante "perfección educativa" que se propone desde los propios maestros hasta todo candidato a presidencia, alcaldía, senado, asamblea, etc., Es así como el tema educativo casi nunca se omite en propuestas para un posible desarrollo colombiano, sin embargo año tras año la educación no parece mejorar.

Para entender la Situación educativa en Colombia, es importante destacar elementos como qué se entiende como "sistemas Educativos". En el texto "Los sistemas educativos en sentido estricto: estructuras, procesos y tendencias" Antonio Viñao ( 2002) plantea que éstos son una red de establecimientos educativos que encuadran, clasifican y alcanzan a toda la población y para establecer el objeto del sistema educativo se establece una organización que es cíclica, es decir, como personas sociales estamos inmersos dentro de un proceso educativo que nunca acaba. Esta decisión se lleva a cabo por cada persona, pero no solo eso; el sistema determina quién ingresa y quién no al sistema educativo.

Así mismo otro elemento es la escuela la cual no es una institución creada por la voluntad política de un gobierno que a partir de una determinada ideología instituyera sus aparatos de dominación desde los cuales ejercería el poder. Tampoco fueron el sistema jurídico, ni la legislación los que definieron la escuela, ni tampoco la evolución de la conciencia al volverse más humanitaria, se pensó en la instrucción

${ }^{1}$ Estudiante de séptimo semestre del Departamento de Biología, Universidad Pedagógica Nacional, Bogotá. 
de masas; fueron otras disciplinas que permitieron atraparla en conceptos. La escuela fue un medio que atrapó y articuló enunciados dispersos: la patria, la luz, la agricultura, entre otros.

Actualmente la escuela se considera como un espacio de interrelación entre el maestro y el alumno principalmente, en la que influyen muchos otros factores. Además de ser el espacio donde se llevan a cabo procesos de creación y distribución de conocimientos, también es un espacio en el que se forman ciudadanos porque en la escuela se viven hechos que son un aprendizaje de la vida en sociedad (Chona, 1998), por ejemplo las relaciones de poder entre maestros y estudiantes; relaciones de poder, entendidos no como hechos de autoritarismo, ni como un objeto que se quita o se toma, sino como un flujo que está en todas partes, en este caso en la escuela, que se relaciona con las decisiones y que no tiene intención, pero que se manifiesta a través de los objetos. Es así, como el maestro es un sujeto político que ejerce un poder, es decir, que el maestro, desde la manera como se concibe a sí mismo, así como su quehacer, construye mundos posibles, legitima y deslegitima proyectos políticos, construye imaginarios y posiciona a otros sujetos ante la realidad. De la manera como interprete esta realidad y se posicione ante ella, dependerá también la posición que asuman los sujetos que lo escuchan y los modelos que se les brinda. (González, 2005).

Por otra parte, es importante hacer un breve recorrido histórico acerca de procesos educativos relevantes en los últimos años en Colombia, para lograr hacer la reflexión sobre la situación educativa en este país, antes, ahora y unas proyecciones a futuro.

Hacia los años 50 el propósito de la educación era disminuir el analfabetismo a partir de la incorporación de las personas en la escuela básica; para estos momentos se piensa que el desarrollo que tiene que ver con la industrialización sólo se podía posibilitar a través de la educación, aspecto que se pensó desde la perspectiva de planeación desde la psicología, disciplina que se creó como lo plantea Foucault (1974) en la verdad y las formas jurídicas, desde la agrupación de los saberes que van de la mano con la práctica, apareciendo las disciplinas y las ciencias que constituyeron al ser humano como objeto de estudio, constituyendo nuevos objetos y nuevos sujetos como los psicólogos que para esta época plantearon la educación desde una perspectiva del conductismo.

Además la educación se pensó desde la elaboración de unos objetivos, que estaban intervenidos por la economía y con ello los organismos internacionales como la CEPAL, la UNESCO y la OEI, que proponían qué enseñar, para qué enseñar y cómo enseñar. Así mismo para este entonces el Ministerio de Instrucción Pública era el encargado de las relaciones con organismos internacionales para lo concerniente a la planeación educativa en Colombia, sabiendo así qué manuales para maestros se deberían usar para el objetivo de desarrollo.

Del mismo modo hacia lo años 50 y 70 se establece una modernización educativa, en la que se buscaba la legislación de los planes de estudio, una reforma en la enseñanza de las ciencias, una organización de los colegios. No sólo se buscaba la verdadera función del maestro, sino también el aumento de la cobertura y la expansión de la escuela, hecho que si fue posible para ese entonces. 
Hacia los años 80 hay un declive de esta expansión, debido a la resistencia por parte de los maestros que crean el movimiento pedagógico, el cual produce un giro estratégico en la educación. Se reelaboró el discurso educativo desde una mirada del neoliberalismo y la globalización, pensando la escuela desde una perspectiva de "escuela competitiva", en la que lo más importante es la competencia; discurso que tiene que ver con la empresa (oferta/demanda). La competencia se piensa desde una mirada de la equidad respecto a la calidad de vida de las personas, apoyadas en el desarrollo humano y el desarrollo sostenible (discurso económico) y de esta forma se habla de unas necesidades básicas (consumo), pera también aparecen en la educación las necesidades básicas para el aprendizaje, en donde lo más importante es cómo se incorporan las personas al mundo laboral, lo cual se mide a través de la evaluación, discurso que hoy en día aún sigue vigente. Es así como la educación, tal como la entienden hoy las agencias internacionales de desarrollo, adquiere valor sólo en la medida en que se conecte con los sistemas globales de producción o, lo que es lo mismo, si entra en el juego del incremento de las competencias económicas, sólo matizada con el fortalecimiento de la democracia representativa. (Boom, 2004)

Conociendo un poco acerca de hechos históricos, también es importante mencionar indicadores económicos de calidad y de cobertura tomados de los documentos de Misión de Ciencia Educación y Desarrollo (1994) y el Salto Educativo (1994). Como por ejemplo que en Colombia se invierte menos del $0.4 \%$ en investigación, esto tal vez debido al desinterés del estado por contribuir en la ciencia, ya que existen los recursos, en comparación con otros países que invierten más del 4\% del PBI en investigación, ó que la tasa de analfabetismo en Colombia es del 13\%, en comparación con otros países en donde este hecho ya no existe.

En lo concerniente a la calidad educativa en Colombia los estudiantes reciben 680 horas de clase al año, esto es un promedio de 3 a 5 horas diarias, en comparación con otros países como Estados Unidos que da 1.100 horas o Japón que da 1.500 horas. Además a una persona que haya asistido a una Normal, se le permite ejercer como profesor de los primeros grados escolares.

El gobierno preocupado por bajar las estadísticas del analfabetismo, trata de incrementar el número de bachilleres, profesionales y "doctores". Se esfuerzan entonces por implementar políticas que coincidan con las "exigencias de un mundo globalizado" y que respondan al concepto de educación de la actualidad, derivado de estas tres fuentes: el discurso de los organismos internacionales, las elaboraciones del campo de la investigación educativa internacional y los discursos de la educación provenientes de los paradigmas clásicos de las ciencias de la Educación y las teorías del currículo (Boom, 2004).

De lo que se trata entonces, es de competir (nosotros, un país subdesarrollado que poco a poco se ha ido ganando los primeros lugares en desempleo, pobreza y violencia) con los países que históricamente han estado en la vanguardia de la economía, la ciencia y la tecnología. A partir de esto los esfuerzos se dirigen a mejorar la calidad de la enseñanza en Colombia, discursos que tratan conceptos como la eficiencia, la productividad, la equidad, la calidad, pero todo en función de los intereses económicos y políticos de las elites internacionales.

Pero a veces este discurso parece ser simplista y la enseñanza se concibe como un proceso técnico que no es para nada reflexivo y se asimila la calidad con la consecución de unos 
"niveles" preestablecidos, por medio de un sistema de supervisión, inspección y control" (Carr, 1993).

La OEI plantea que no sólo el sistema escolar no está transmitiendo adecuadamente los aprendizajes que promete, como muestran las diversas formas de medición de los niveles de aprendizaje que se están implementando en países de la región, sino que estos aprendizajes suelen ser insuficientes o inadecuados para los desafíos que los jóvenes tienen en tanto jóvenes y tendrán por delante como futuros adultos" (OEI, 1994). Es así como para el año 2007 esto sigue en vigencia, además las políticas que se implantan día tras día como los estándares curriculares, bajo la idea de que todos debemos aprender lo mismo para asumir la idea de "persona competente y productiva", hacen parte de la real situación educativa en Colombia.

La ONU organismo internacional afirma que: “... existen cinco "potenciadores" de desarrollo del recurso humano: educación; salud y nutrición; medio ambiente; empleo, y la libertad económica y política. Estos potenciadores están conectados entre sí y son interdependientes, pero la educación constituye la base de todos los demás y es un factor esencial en el mejoramiento de la salud y la nutrición, la preservación de la calidad del medio ambiente, la expansión y mejoramiento de los recursos laborales, y como sustento de la responsabilidad política y económica. Por lo tanto, no debe sorprender que todos los países pongan un gran énfasis en las políticas educativas cuando diseñan sus planes para acelerar el desarrollo" (Hallak, 1991: 17.). Pero todas estas cosas que se proponen para el mejoramiento de la educación y con ello un mejor desarrollo, se mueven a la par de estrategias e intereses económicos del FMI, BM o BID.

Lo que si es cierto es que la educación Colombiana no será mejor si obedece a las recomendaciones de la CEPAL, La UNESCO o el FMI, que lo que buscan es la eficiencia de las personas dentro de un mundo productivo.

Es así como las políticas educativas en Colombia, se encuentran en la dimensión de la modificación de indicadores numéricos como porcentaje de deserción, número de graduados, cantidad de dinero invertido en relación con el número de estudiantes por maestro, etc. Sin embargo, no se ha pensado en los verdaderos problemas que acrecientan a la sociedad colombiana y lo más conveniente sería dejar a un lado las máscaras y la imagen ante otros países y empezar de verdad a pensarnos como sujetos sociales, ya que entre las prácticas y los saberes sociales el sujeto se va constituyendo mediante su inserción en lo público a través del universo simbólico de significados, leyes y normas que el Estado promueve, y mediatizado por las relaciones de poder en que se encuentra envuelto. En otras palabras, los dominios o campos en los cuales se expresa el sujeto social se dan en lo público, en donde se manifiesta lo visible, se expresan las identidades, el espacio donde se habita y se interactúa; en lo simbólico dado por el universo de significados que el sujeto comparte en sus diversas comunidades de prácticas; y en las relaciones de poder que el Estado, a través de sus diversas instituciones y organizaciones, abroga o reglamenta códigos jurídicos, normas morales o prescripciones o convenciones sociales (Gómez, 2000).

Si bien es cierto dependemos de otros agentes, pero podemos proponer soluciones estratégicas en este caso para la situación educativa en Colombia.

Pero ¿cuál es la situación del maestro en estos aspectos y qué se puede proponer desde el aula en donde nos encontramos? 
Revoluciones como la tecnológica han situado al maestro como administrador del currículo, es decir, gestores fundamentales de la aplicación de los contenidos de una disciplina. Consecuencia de esto es la organización educativa que muchas veces no tiene en cuenta la realidad propia de cada lugar en donde será aplicado el currículo, las necesidades en vez de ser una prioridad fundamental se convierten en algo alejado, ya que las personas que se encargan del diseño de planes de estudio conocen de cultura universal pero no de la nuestra, ellos indican qué se debe enseñar, en dónde, cuándo y cómo.

Así mismo en Colombia se han creado políticas, leyes que determinan la organización, estructura, planes educativos y otros concernientes a la educación, de ahí que algunos maestros podamos ejercer inteligentemente, es decir, conociendo y aplicando nuestros derechos y deberes constitucionales.

Pero uno de los grandes problemas que se vive actualmente respecto al papel del maestro, es el desconocimiento de nuestra cultura ¿quiénes somos? ¿Cómo obramos? ¿Cómo conocemos?. Gabriel García Márquez, en Misión de Ciencia, Educación y Desarrollo, plantea que nosotros los colombianos tenemos dos dones naturales que han ayudado a suplir los vacíos de nuestra identidad cultural y social: el de la creatividad y el ascenso personal, talentos precolombianos que también han ayudado a desenvolvernos en cualquier medio y aprender cualquier oficio y que pueden ser parte de la revolución educativa Colombiana.

Por otra parte, en un aula de clase, el maestro se encuentra acostumbrado a ser monotemático, es decir, en centrarse en un solo tema, sabiendo que los niños de ahora desarrollan varias habilidades al mismo tiempo, entonces ¿por qué no aprovechar estas habilidades para abarcar temas en clase, estimulando y desarrollando la creatividad de niños y jóvenes?.

En varios informes, como el informe Atlántida respecto a un trabajo etnográfico realizado, se constató que los niños y jóvenes de hoy en día no van al colegio a aprender como muchos creemos, más bien dijeron que ellos van a hacer vida social, a compartir con el par y que muchas veces mienten acerca de sus condiciones por temor. Como maestros debemos apoyarnos en estos estudios para saber qué enseñar y de qué forma.

En conclusión, la situación educativa en Colombia es un asunto que sí tiene salida, como maestros en formación también podemos aportar muchas ideas, cambiando desde el aula de clase las perspectivas que se tienen acerca de cómo se enseña y se aprende, pensando en qué clase de sujetos y de sociedad se quiere formar, desde la perspectiva de mundo globalizado en la que nos encontramos inmersos; formando sujetos políticos, autónomos y responsables frente a la realidad que nos acrecienta; no dando órdenes o instrucciones acerca de cómo obrar frente a la vida, que sin duda alguna brindan algunos maestros que pensando en la incorporación laboral de sus alumnos a futuro, se encargan de dar todo a manera de recetas, instrucciones y órdenes que según ellos más adelante les "servirán para su trabajo" en empresas, auge laboral al que la mayoría de personas quieren pertenecer, bajo el lema de competitividad, eficiencia y productividad. 


\section{Bibliografía}

- Carr, W. (1993). Calidad de la Enseñanza e Investigación - Acción. Diada Editora, Sevilla. 194 p.

- Foucault, M. (1974). La verdad y las formas jurídicas. Memorias 5 conferencias. p. 68. Río de Janeiro.

- Gómez, J. H. (2002). La construcción del conocimiento social en la escuela. Universidad Distrital, Centro de Investigaciones, Bogotá.

- González, A.M. (2005). Poder, políticas y política. En: Revista Educación y Ciudad. (Bogotá) № 9.

- Hallack, J. (1991). Invertir en el Futuro. PNUD - IIPE (Instituto Internacional de Planeamiento de la Educación). Tecnos, UNESCO. Madrid.

- Martínez, A. (2004). De la escuela expansiva a la escuela competitiva. Dos modos de modernización en América Latina. Anthropos, España.

- OEI-Organización de Estados Iberoamericanos. Programa Regional de Acciones para el Desarrollo de la Juventud en América Latina. (1994).Resúmenes. VII Conferencia Iberoamericana de Ministros de Juventud. Punta del Este, Uruguay.

- Toranzos, L. (1996). Evaluación y Calidad. En: Revista Iberoamericana de Educación. (Madrid) № 10 .

- Viñao, A. (2002). Sistemas educativos, culturas escolares y reformas: continuidades y cambios. Ediciones Morata. Madrid España. 127 p. 